Research Paper

\title{
Association between SNPs in Long Non-coding RNAs and the Risk of Female Breast Cancer in a Chinese Population
}

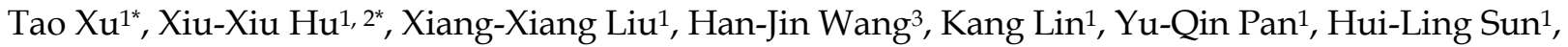 \\ Hong-Xin Peng1, 2, Xiao-Xiang Chen ${ }^{1,2}$, Shu-Kui Wang ${ }^{1 凶}$ and Bang-Shun $\mathrm{He}^{1 \bowtie}$ \\ 1. General Clinical Research Center, Nanjing First Hospital, Nanjing Medical University, Nanjing, China; \\ 2. Medical college, Southeast University, Nanjing, China; \\ 3. Department of General Surgery, Nanjing First Hospital, Nanjing Medical University, Nanjing, China. \\ * These authors contributed equally to this work. \\ $\square$ Corresponding authors: Shu-Kui Wang Email: sk_wang@njmu.edu.cn; Bang-Shun He Email: hebangshun@163.com \\ (c) Ivyspring International Publisher. This is an open access article distributed under the terms of the Creative Commons Attribution (CC BY-NC) license \\ (https://creativecommons.org/licenses/by-nc/4.0/). See http://ivyspring.com/terms for full terms and conditions.
}

Received: 2016.10.24; Accepted: 2016.12.19; Published: 2017.04.09

\begin{abstract}
Long non-coding RNAs (LncRNAs) have been reported to be involved in tumorigenesis and tumor progression. Single nucleotide polymorphisms (SNPs) in the IncRNAs also play a vital role in carcinogenesis. The aim of this study was to assess the relationships between the four selected tagSNPs (rs944289, rs3787016, rs1456315, rs7463708) in the IncRNAs and the risk of female breast cancer in a Chinese population. A case-control study was carried out involving in a total of 439 breast cancer patients and 439 age-matched healthy controls. The genotyping was performed with Sequenom MassARRAY and the expression of estrogen receptor (ER), progesterone receptor (PR) and human epidermal growth factor receptor-2 (HER-2) in tumor tissues was measured by the immunohistochemistry (IHC) assay. We found that rs3787016 TT genotype (adjusted odds ratio $(\mathrm{OR})=1.62,95 \%$ confidence interval $(\mathrm{Cl})=1.09-2.41, P=0.018$ ) was associated with an increased risk of female breast cancer, especially among the patients with premenopausal status (adjusted $\mathrm{OR}=2.55,95 \% \mathrm{Cl}=1.30-4.97, P=0.006$ ). Moreover, a statistically significant increased risk of the rs3787016 TT genotype was observed among the patients with advanced tumor stage (III and IV), poor histological grade (G3-G4), positive lymph node involvement, positive expression of ER and PR and negative expression of HER-2; rs7463708 GT and GT/GG genotype were associated with decreased risk of breast cancer in the subgroup of patients with postmenopausal status (GT versus (vs.) TT: adjusted $\mathrm{OR}=0.67,95 \% \mathrm{Cl}=0.46-0.99$, $P=0.043 ; \mathrm{GT} / \mathrm{GG}$ vs. TT: adjusted $\mathrm{OR}=0.68,95 \% \mathrm{Cl}=0.47-0.98, P=0.041)$ and tumor late-stage (GT vs. TT: adjusted $\mathrm{OR}=0.65,95 \% \mathrm{Cl}=0.43-0.97, P=0.037 ; \mathrm{GT} / \mathrm{GG}$ vs. TT: adjusted $\mathrm{OR}=0.65$, $95 \% \mathrm{Cl}=0.44-0.96, P=0.029)$. In short, $r$ s3787016 TT genotype was associated with increased breast cancer risk and clinicopathologic features of the tumor, especially among premenopausal women.
\end{abstract}

Key words: Breast cancer; LncRNAs; SNPs

\section{Introduction}

Breast cancer $(\mathrm{BC})$ is recognized as the most common malignant tumor and the leading cause of cancer-related death among females all over the world [1]. In China, breast cancer was responsible for around 268,600 new cases and 69,500 deaths in 2015
[2]. Studies have identified environmental factors as the risk for breast cancer, involving reproductive and hormonal factors including a long menstrual history, oral contraceptives use and never having children [1]. Also, genetic backgrounds play a vital role in the 
etiology of breast cancer. Previous researches have reported that a number of genetic variants were associated with the risk of $\mathrm{BC}$ [3-5]. However, the occurrence of $\mathrm{BC}$ is a complex multifactorial process and the molecular mechanism remains largely unclear.

Long non-coding RNAs (LncRNAs) are a new class of regulatory non-coding RNAs with length longer than 200 nucleotides, lacking open reading frame and having no potential protein translation capacity [6]. Recently, many studies have revealed that aberrant expression of lncRNAs was significantly associated with tumorigenesis and tumor progression in different cancer types, indicating the lncRNAs act as proto-oncogene [7] or anti-oncogene [8]. In addition, IncRNAs are considered to be involved in complex pathogenesis of cancers, referring to the levels of epigenome, transcription and post-transcription [9]. LncRNAs have essential roles in multiple biological processes including chromatin remodeling, cell differentiation, cell cycle control, genome rearrangement, dosage compensation, gene imprinting and regulation of gene expression [10]. Study demonstrated that the lncRNA HOTAIR was overexpressed in BC tissues and participated in $B C$ progression [9]. Also, up-regulated lncRNA MALAT1 was detected in lung adenocarcinoma, which predicted metastasis and poor prognosis in early-stage non-small cell lung cancer [11]. Additionally, research has shown that lncRNA MEG3 expression was down-regulated in gastric cancer tissues and cell lines, and was associated with metastasis of gastric cancer by its function as a competing endogenous RNA (ceRNA) of miR-181s to regulate gastric cancer progression [12].

SNPs in lncRNAs may affect the function of target genes through altering the process of splicing and stability of mRNA conformation, leading to the modification of their interacting partners [13]. To date, the susceptibility of lncRNA SNPs to cancer risk have been investigated by numerous researches, such as lncRNA HOTAIR rs920778 polymorphism cause HOTAIR up-regulated among T allele carriers, which enhancing esophageal squamous cell carcinoma (ESCC)[14] and cervical cancer [15] risk in a Chinese population. However, Bayram et al. [16] reported that HOTAIR rs920778 CC genotype significantly increased the $\mathrm{BC}$ risk in a Turkish population. In addition, it was identified that IncRNA HULC rs7763881 may decrease the risk of HBV-related hepatocellular carcinoma in a Chinese population [17]. Subsequently, genome-wide association studies (GWAS) have identified that the C allele carriers of rs12325489 in the lincRNA-ENST00000515084 are associated with increased BC risk [18].
Based on the above backgrounds, the SNPs in the lncRNAs were the susceptibility of $B C$, and could be invested as biomarkers for the risk of BC. To date, there is no research to evaluate the susceptibility of lncRNA PTCSC3 rs944289, IncRNA POLR2E rs3787016, lncRNA PRNCR1 rs1456315 and lncRNA PRNCR1 rs7463708 to BC risk. Therefore, in the current study, we selected these four tagSNPs in the lncRNAs and evaluated the relationships between the four SNPs and the risk of $\mathrm{BC}$ in a Chinese female population.

\section{Materials and Methods}

\section{Study subjects}

A total of 878 age-matched female subjects divided into case cohort with $439 \mathrm{BC}$ patients and health cohort with 439 cancer-free individuals were enrolled in this population-based case-control study. All the patients were genetically unrelated and consecutively recruited starting from January 2008 to January 2016 in Nanjing First Hospital, Nanjing Medical University, China. Meanwhile, the health controls were randomly collected in the same hospital for their routine physical checkup at the same time period. For the cases and controls, a pretested questionnaire was used to record clinical information of each individual, such as tobacco smoking, alcohol consumption and other cancer history. Owing to less than ten individuals have the history of smoking and drinking, which may be attributed to the life style of Chinese female, and considering the very small size of participants has these two environmental factors, finally we adjusted inclusion criteria of cases as follows: (1) subjects were histologically diagnosed with primary BC; (2) with no history of smoking and drinking; (3) with no evidence of personal or family history of cancer. Selection criteria for controls included no prior history of cancer or other malignant conditions and without history of smoking and drinking. All participants have given written informed consents, and this study was approved by the Institutional Review Board of the Nanjing First Hospital.

\section{SNPs genotyping}

We collected these blood samples from each individual after their admission to the hospital. The whole blood samples of all participants collected in a test tube containing EDTA were used for genotyping assay. Genomic DNA was isolated from peripheral white blood and concentrated by using GoldMag-Mini Whole Blood Genomic DNA Purification Kit according to the manufacturer's directions (GoldMag Co. Ltd. Xian, China). The extracted DNA was stored at $-80^{\circ} \mathrm{C}$ until use. We 
adopted the spectrometry (DU530 UV/vis spectrophotometer, Beckman Instruments, Fullerton, CA, USA) to detect DNA purity. Sequenom MassARRAY Assay Design 3.0 Software was used to design Multiplexed SNP MassEXTEND assay [19]. PCR and extension primers were designed by Sequenom, Inc. Assay Design. EXO-SAP was used to digest PCR-amplified DNA, and then mixed the primer extended by IPLEX chemistry, desalted using Clean Resin (Sequenom) and spotted onto Spectrochip matrix chips. Finally, results were detected by Mass Spectrometer. All samples were genotyped by Sequenom MassARRAY RS1000 according to the manufacturer's protocol. The final data was managed and analysed by Sequenom Typer 4.0 Software $[19,20]$.

\section{Immunohistochemistry (IHC) assay}

The immunohistochemistry (IHC) assay was applied to evaluate the expression of estrogen receptor (ER), progesterone receptor (PR) and the human epidermal growth factor receptor 2 (HER-2) in paraffin-embedded tumor tissue[21]. The immunohistochemical analysis was conducted following the instructions inside the kit. The monoclonal rabbit ER, PR and HER-2 antibody used in this study were purchased from Spring Bioscience (Pleasanton, CA, USA).

\section{Statistical analysis}

Hardy-Weinberg equilibrium (HWE) was assessed by a goodness of fit chi-square test among the healthy controls to compare the observed genotype frequencies with the expected ones. The two-sided $\chi^{2}$ test and independent $t$ test were used to compare the selected variables between BC patients and healthy controls. Associations of the four SNPs with $\mathrm{BC}$ risks were estimated by a logistic regression model with odds ratios (ORs) and 95\% confidence intervals (CIs), corresponding $p$ values after adjustment for age and menopausal status. All statistical analysis was performed by using SPSS 23.0 for Windows (SPSS, Chicago, IL) and the $P$ value < 0.05 was considered to be statistically significant.

\section{Results}

A total of 878 age-matched Chinese women subjects (439 BC patients and 439 healthy controls) were enrolled in this population-based case-control study to investigate the potential association between the four tagSNPs (rs944289, rs3787016, rs1456315, rs7463708) in the lncRNAs and BC risk. Clinicopathological features of patients with $\mathrm{BC}$ and healthy controls were summarized in Table 1, there were no statistically significant differences in age and menopausal status (mean age of patients vs. controls: $52.89 \pm 10.78$ years vs. $52.95 \pm 10.89$ years, $P=0.933$; number of postmenopausal cases vs. controls: $241 \mathrm{vs.}$ $229, P=0.417)$. The observed genotype frequencies of the four SNPs in healthy controls were no significant deviations from the Hardy-Weinberg equilibrium (HWE) $(P=0.078$ for rs944289, $P=0.144$ for rs3787016, $P=0.167$ for $\operatorname{rs} 1456315$ and $P=0.142$ for rs7463708, respectively).

Table 1. Clinicopathological features of patients with breast cancer and healthy controls

\begin{tabular}{|c|c|c|c|}
\hline Variables & Cases, n (\%) & Controls, n (\%) & $P$ value \\
\hline Total participants & 439 & 439 & \\
\hline Age(Mean $\pm S D$, years) & $52.89 \pm 10.78$ & $52.95 \pm 10.89$ & 0.933 \\
\hline Menopausal status & & & 0.417 \\
\hline Premenopausal & 198(45.10\%) & $210(47.84 \%)$ & \\
\hline Postmenopausal & $241(54.90 \%)$ & $229(52.16 \%)$ & \\
\hline \multicolumn{4}{|l|}{ Tumor stage } \\
\hline $0-\mathrm{II}$ & $306(69.70 \%)$ & & \\
\hline III-IV & $133(30.30 \%)$ & & \\
\hline \multicolumn{4}{|l|}{ Tumor grade } \\
\hline G1-G2 & $317(72.21 \%)$ & & \\
\hline G3-G4 & $122(27.79 \%)$ & & \\
\hline \multicolumn{4}{|c|}{ Lymph node involvement } \\
\hline Negative & $211(48.06 \%)$ & & \\
\hline Positive & $228(51.94 \%)$ & & \\
\hline \multicolumn{4}{|l|}{ ER } \\
\hline Negative & $166(37.81 \%)$ & & \\
\hline Positive & $273(62.19 \%)$ & & \\
\hline \multicolumn{4}{|l|}{ PR } \\
\hline Negative & $205(46.70 \%)$ & & \\
\hline Positive & $234(53.30 \%)$ & & \\
\hline \multicolumn{4}{|l|}{ HER-2 } \\
\hline Negative & $92(20.96 \%)$ & & \\
\hline Positive & $347(79.04 \%)$ & & \\
\hline
\end{tabular}

The genotypes and alleles distribution of the four tagSNPs in BC patients and healthy controls are presented in Table 2. Logistic regression analysis revealed that the rs3787016 TT homozygote (adjusted OR $=1.62,95 \%$ CI: 1.09-2.41, $P=0.018$ ) was associated with increased risk of $\mathrm{BC}$ when compared with the wild-type CC homozygote. Also, a borderline significantly increased risk was observed in the $\mathrm{T}$ allele of rs3787016 (adjusted OR $=1.21,95 \% \mathrm{CI}$ : 1.00-1.46, $P=0.052$ ) for $B C$ when compared with the $C$ allele. However, no statistically significant association between rs944289, rs1456315 and rs7463708 and the risk of BC was observed among all participants, as shown in Table 2. 
Table 2. Genotypes and allele frequencies of the four SNPs between patients with breast cancer and healthy controls

\begin{tabular}{|c|c|c|c|c|c|c|}
\hline Genotype & Cases, n(\%) & Controls, $\mathrm{n}(\%)$ & Crude OR $(95 \% \mathrm{CI})$ & $P$ value & Adjusted OR $(95 \% \mathrm{CI})^{\mathrm{a}}$ & $P$ value \\
\hline \multicolumn{7}{|l|}{ rs944289 } \\
\hline $\mathrm{CC}$ & $127(28.93)$ & $115(26.20)$ & Reference & & Reference & \\
\hline CT & $229(52.16)$ & 237(53.99) & $0.88(0.64,1.19)$ & 0.400 & $0.88(0.64,1.20)$ & 0.402 \\
\hline TT & $83(18.91)$ & 87(19.82) & $0.86(0.58,1.28)$ & 0.465 & $0.86(0.58,1.28)$ & 0.453 \\
\hline $\mathrm{CT} / \mathrm{TT}$ & $312(71.07)$ & $324(73.80)$ & $0.87(0.65,1.17)$ & 0.365 & $0.87(0.65,1.17)$ & 0.368 \\
\hline \multicolumn{7}{|l|}{ Allele } \\
\hline $\mathrm{C}$ & $483(55.01)$ & $467(53.19)$ & Reference & & Reference & \\
\hline $\mathrm{T}$ & $395(44.99)$ & $411(46.81)$ & $0.93(0.77,1.12)$ & 0.444 & $0.93(0.77,1.12)$ & 0.442 \\
\hline \multicolumn{7}{|l|}{ rs3787016 } \\
\hline $\mathrm{CC}$ & $137(31.21)$ & $149(33.94)$ & Reference & & Reference & \\
\hline $\mathrm{TC}$ & $209(47.61)$ & $226(51.48)$ & $1.01(0.75,1.36)$ & 0.970 & $1.02(0.76,1.38)$ & 0.894 \\
\hline TT & $93(21.18)$ & $64(14.58)$ & $1.58(1.07,2.34)$ & 0.023 & $1.62(1.09,2.41)$ & 0.018 \\
\hline $\mathrm{TC} / \mathrm{TT}$ & $302(68.79)$ & $290(66.06)$ & $1.13(0.85,1.50)$ & 0.388 & $1.13(0.85,1.51)$ & 0.384 \\
\hline \multicolumn{7}{|l|}{ Allele } \\
\hline $\mathrm{C}$ & $483(55.01)$ & $524(59.68)$ & Reference & & Reference & \\
\hline $\mathrm{T}$ & $395(44.99)$ & $354(40.32)$ & $1.21(1.01,1.46)$ & 0.048 & $1.21(1.00,1.46)$ & 0.052 \\
\hline \multicolumn{7}{|l|}{ rs1456315 } \\
\hline $\mathrm{AA}$ & $234(53.30)$ & $244(55.58)$ & Reference & & Reference & \\
\hline GA & $165(37.59)$ & $159(36.22)$ & $1.08(0.82,1.44)$ & 0.584 & $1.08(0.81,1.43)$ & 0.607 \\
\hline GG & $40(9.11)$ & $36(8.20)$ & $1.16(0.71,1.88)$ & 0.552 & $1.16(0.71,1.88)$ & 0.555 \\
\hline GA/GG & $205(46.70)$ & 195(44.42) & $1.10(0.84,1.43)$ & 0.498 & $1.09(0.84,1.43)$ & 0.511 \\
\hline \multicolumn{7}{|l|}{ Allele } \\
\hline A & 633(72.10) & 647(73.69) & Reference & & Reference & \\
\hline G & $245(27.90)$ & $231(26.31)$ & $1.08(0.88,1.34)$ & 0.452 & $1.08(0.88,1.34)$ & 0.460 \\
\hline \multicolumn{7}{|l|}{ rs7463708 } \\
\hline TT & $209(47.61)$ & $184(41.91)$ & Reference & & Reference & \\
\hline GT & $190(43.28)$ & $211(48.06)$ & $0.79(0.60,1.05)$ & 0.103 & $0.79(0.60,1.05)$ & 0.102 \\
\hline GG & $40(9.11)$ & $44(10.02)$ & $0.80(0.50,1.28)$ & 0.355 & $0.81(0.50,1.30)$ & 0.380 \\
\hline GT/GG & $230(52.39)$ & 255(58.09) & $0.79(0.61,1.04)$ & 0.090 & $0.79(0.61,1.04)$ & 0.090 \\
\hline \multicolumn{7}{|l|}{ Allele } \\
\hline $\mathrm{T}$ & $608(69.25)$ & $579(65.95)$ & Reference & & Reference & \\
\hline G & $270(30.75)$ & $299(34.05)$ & $0.86(0.70,1.05)$ & 0.139 & $0.86(0.70,1.05)$ & 0.139 \\
\hline
\end{tabular}

aAdjusted by age and menopausal status in logistic regression analysis.

The bold values indicate statistically significant data

Table 3. Stratified effects of polymorphisms in IncRNAs on breast cancer risk by menopausal status

\begin{tabular}{|c|c|c|c|c|c|c|}
\hline \multirow[t]{2}{*}{ Genotype } & \multicolumn{2}{|l|}{ Premenopausal } & \multirow[t]{2}{*}{$P$ value } & \multicolumn{2}{|l|}{ Postmenopausal } & \multirow[t]{2}{*}{$P$ value } \\
\hline & Patients/controls & $\mathrm{OR}(95 \% \mathrm{CI})^{\mathrm{a}}$ & & Patients/controls & $\mathrm{OR}(95 \% \mathrm{CI})^{\mathrm{a}}$ & \\
\hline \multicolumn{7}{|l|}{ rs944289 } \\
\hline $\mathrm{CC}$ & $55 / 54$ & Reference & & $72 / 61$ & Reference & \\
\hline $\mathrm{CT}$ & $110 / 112$ & $0.97(0.61,1.53)$ & 0.879 & $119 / 125$ & $0.81(0.53,1.23)$ & 0.319 \\
\hline TT & $33 / 44$ & $0.74(0.41,1.33)$ & 0.312 & $50 / 43$ & $0.97(0.56,1.66)$ & 0.903 \\
\hline $\mathrm{CT} / \mathrm{TT}$ & $143 / 156$ & $0.90(0.58,1.40)$ & 0.638 & $169 / 168$ & $0.85(0.57,1.27)$ & 0.428 \\
\hline \multicolumn{7}{|l|}{ rs3787016 } \\
\hline $\mathrm{CC}$ & $61 / 65$ & Reference & & $76 / 84$ & Reference & \\
\hline $\mathrm{TC}$ & $97 / 128$ & $0.79(0.51,1.23)$ & 0.304 & $112 / 98$ & $1.23(0.81,1.87)$ & 0.324 \\
\hline TT & $40 / 17$ & $2.55(1.30,4.97)$ & 0.006 & $53 / 47$ & $1.25(0.75,2.08)$ & 0.387 \\
\hline $\mathrm{TC} / \mathrm{TT}$ & $137 / 145$ & $1.01(0.66,1.54)$ & 0.970 & $165 / 145$ & $1.24(0.85,1.82)$ & 0.272 \\
\hline \multicolumn{7}{|l|}{ rs1456315 } \\
\hline AA & $99 / 113$ & Reference & & $135 / 131$ & Reference & \\
\hline GA & $81 / 78$ & $1.18(0.78,1.78)$ & 0.431 & $84 / 81$ & $0.99(0.67,1.47)$ & 0.971 \\
\hline GG & $18 / 19$ & $1.08(0.54,2.17)$ & 0.832 & $22 / 17$ & $1.24(0.63,2.44)$ & 0.540 \\
\hline GA/GG & $99 / 97$ & $1.16(0.79,1.72)$ & 0.444 & $106 / 98$ & $1.04(0.72,1.49)$ & 0.855 \\
\hline \multicolumn{7}{|l|}{ rs7463708 } \\
\hline $\mathrm{TT}$ & $85 / 87$ & Reference & & $124 / 97$ & Reference & \\
\hline GT & $93 / 100$ & $0.96(0.63,1.44)$ & 0.826 & $97 / 111$ & $0.67(0.46,0.99)$ & 0.043 \\
\hline GG & $20 / 23$ & $0.89(0.46,1.74)$ & 0.733 & $20 / 21$ & $0.74(0.38,1.43)$ & 0.366 \\
\hline GT/GG & $113 / 123$ & $0.94(0.64,1.39)$ & 0.760 & $117 / 132$ & $0.68(0.47,0.98)$ & 0.041 \\
\hline
\end{tabular}

aAdjusted by age

The results with significant difference are in bold 
Table 4. Stratified effects of SNPs in IncRNAs on breast cancer risk by the pathological characteristics of patients

\begin{tabular}{|c|c|c|c|c|c|c|c|c|c|c|c|c|c|c|c|c|c|c|c|}
\hline \multirow[t]{2}{*}{ Genotype } & \multirow[t]{2}{*}{ Co } & \multicolumn{2}{|c|}{ Stage(0-II) } & \multirow[t]{2}{*}{$\begin{array}{l}P \\
\text { value }^{a}\end{array}$} & \multicolumn{2}{|c|}{ Stage(III-IV) } & \multirow[t]{2}{*}{$\begin{array}{l}P \\
\text { value }^{a}\end{array}$} & \multicolumn{2}{|c|}{ Grade(G1-G2) } & \multirow[t]{2}{*}{$\begin{array}{l}P \\
\text { value }^{\text {a }}\end{array}$} & \multicolumn{2}{|c|}{ Grade(G3-G4) } & \multirow[t]{2}{*}{$\begin{array}{l}P \\
\text { value }^{\mathrm{a}}\end{array}$} & \multicolumn{2}{|c|}{$\begin{array}{l}\text { Lymph node } \\
\text { involvement(-) }\end{array}$} & \multirow[t]{2}{*}{$\begin{array}{l}P \\
\text { value }^{\text {a }}\end{array}$} & \multicolumn{2}{|c|}{$\begin{array}{l}\text { Lymph node } \\
\text { involvement(+) }\end{array}$} & \multirow[t]{2}{*}{$\begin{array}{l}P \\
\text { value }\end{array}$} \\
\hline & & $\mathrm{Ca}$ & OR(95\%CI) & & $\mathrm{Ca}$ & OR(95\%CI) & & $\mathrm{Ca}$ & OR(95\%CI) & & $\mathrm{Ca}$ & OR(95\%CI) & & $\mathrm{Ca}$ & $\mathrm{OR}(95 \% \mathrm{CI})$ & & $\mathrm{Ca}$ & $\mathrm{OR}(95 \% \mathrm{CI})$ & \\
\hline \multicolumn{20}{|l|}{ rs944289 } \\
\hline $\mathrm{CC}$ & 115 & 92 & Reference & & 35 & Reference & & 92 & Reference & & 35 & Reference & & 62 & Reference & & 65 & Reference & \\
\hline $\mathrm{CT}$ & 237 & 159 & $0.84(0.60,1.18)$ & 0.314 & 70 & $0.97(0.61,1.53)$ & 0.879 & 166 & $0.88(0.62,1.23)$ & 0.440 & 63 & $0.87(0.55,1.40)$ & 0.568 & 110 & $0.86(0.59,1.26)$ & 0.442 & 119 & $0.89(0.61,1.29)$ & 0.536 \\
\hline TT & 87 & 55 & $0.79(0.51,1.23)$ & 0.300 & 28 & $1.06(0.60,1.88)$ & 0.851 & 59 & $0.85(0.55,1.31)$ & 0.454 & 24 & $0.91(0.50,1.64)$ & 0.741 & 39 & $0.84(0.51,1.37)$ & 0.478 & 44 & $0.89(0.55,1.44)$ & 0.633 \\
\hline $\mathrm{CT} / \mathrm{TT}$ & 324 & 214 & $0.83(0.60,1.14)$ & 0.252 & 98 & $0.99(0.64,1.54)$ & 0.954 & 225 & $0.87(0.63,1.20)$ & 0.393 & 87 & $0.88(0.56,1.37)$ & 0.570 & 149 & $0.85(0.59,1.22)$ & 0.382 & 163 & $0.89(0.62,1.27)$ & 0.520 \\
\hline \multicolumn{20}{|l|}{ rs3787016 } \\
\hline $\mathrm{CC}$ & 149 & 103 & Reference & & 34 & Reference & & 101 & Reference & & 36 & Reference & & 70 & Reference & & 67 & Reference & \\
\hline $\mathrm{TC}$ & 226 & 145 & $0.95(0.69,1.32)$ & 0.769 & 64 & $1.24(0.78,1.98)$ & 0.371 & 151 & $1.00(0.72,1.39)$ & 0.994 & 58 & $1.09(0.68,1.74)$ & 0.716 & 98 & $0.94(0.64,1.36)$ & 0.723 & 111 & $1.11(0.77,1.61)$ & 0.580 \\
\hline TT & 64 & 58 & $1.34(0.86,2.07)$ & 0.194 & 35 & $2.57(1.45,4.56)$ & 0.001 & 65 & $1.53(1.00,2.36)$ & 0.053 & 28 & $1.92(1.07,3.46)$ & 0.029 & 43 & $1.47(0.90,2.38)$ & 0.121 & 50 & $1.79(1.11,2.89)$ & 0.016 \\
\hline $\mathrm{TC} / \mathrm{TT}$ & 290 & 203 & $1.02(0.75,1.39)$ & 0.911 & 99 & $1.48(0.96,2.29)$ & 0.080 & 216 & $1.10(0.81,1.50)$ & 0.549 & 86 & $1.23(0.79,1.90)$ & 0.361 & 141 & $1.03(0.73,1.46)$ & 0.858 & 161 & $1.23(0.87,1.75)$ & 0.239 \\
\hline \multicolumn{20}{|l|}{ rs1456315 } \\
\hline AA & 244 & 157 & Reference & & 77 & Reference & & 164 & Reference & & 70 & Reference & & 112 & Reference & & 122 & Reference & \\
\hline GA & 159 & 119 & $1.16(0.85,1.59)$ & 0.344 & 46 & $0.90(0.59,1.37)$ & 0.620 & 122 & $1.13(0.83,1.54)$ & 0.451 & 43 & $0.94(0.61,1.45)$ & 0.794 & 77 & $1.05(0.74,1.50)$ & 0.781 & 88 & $1.09(0.78,1.54)$ & 0.610 \\
\hline GG & 36 & 30 & $1.29(0.76,2.19)$ & 0.340 & 10 & $0.87(0.41,1.83)$ & 0.707 & 31 & $1.28(0.76,2.16)$ & 0.350 & 9 & $0.86(0.39,1.88)$ & 0.708 & 22 & $1.33(0.75,2.37)$ & 0.335 & 18 & $1.01(0.55,1.85)$ & 0.982 \\
\hline GA/GG & 195 & 149 & $1.19(0.89,1.60)$ & 0.248 & 56 & $0.90(0.61,1.33)$ & 0.588 & 153 & $1.16(0.86,1.55)$ & 0.330 & 52 & $0.93(0.62,1.40)$ & 0.735 & 99 & $1.11(0.80,1.54)$ & 0.552 & 106 & $1.08(0.78,1.49)$ & 0.657 \\
\hline \multicolumn{20}{|l|}{ rs7463708 } \\
\hline TT & 184 & 139 & Reference & & 70 & Reference & & 148 & Reference & & 61 & Reference & & 98 & Reference & & 111 & Reference & \\
\hline GT & 211 & 138 & $0.87(0.64,1.18)$ & 0.357 & 52 & $0.65(0.43,0.97)$ & 0.037 & 138 & $0.81(0.59,1.09)$ & 0.164 & 52 & $0.75(0.49,1.14)$ & 0.172 & 92 & $0.82(0.58,1.16)$ & 0.261 & 98 & $0.77(0.55,1.07)$ & 0.120 \\
\hline GG & 44 & 29 & $0.89(0.53,1.49)$ & 0.653 & 11 & $0.65(0.32,1.33)$ & 0.240 & 31 & $0.89(0.53,1.48)$ & 0.645 & 9 & $0.61(0.28,1.33)$ & 0.212 & 21 & $0.90(0.51,1.60)$ & 0.723 & 19 & $0.72(0.40,1.31)$ & 0.282 \\
\hline GT/GG & 255 & 167 & $0.87(0.65,1.17)$ & 0.344 & 63 & $0.65(0.44,0.96)$ & 0.029 & 169 & $0.82(0.61,1.09)$ & 0.174 & 61 & $0.72(0.48,1.08)$ & 0.116 & 113 & $0.83(0.60,1.16)$ & 0.276 & 117 & $0.76(0.55,1.04)$ & 0.090 \\
\hline
\end{tabular}

aAdjusted by age and menopausal status

The bold values indicate statistically significant data

Table 5. Stratified effects of SNPs in IncRNAs on breast cancer risk by the expression of ER,PR and HER-2

\begin{tabular}{|c|c|c|c|c|c|c|c|c|c|c|c|c|c|c|c|c|c|c|c|}
\hline \multirow[t]{2}{*}{ Genotype } & \multirow[t]{2}{*}{ Co } & \multicolumn{2}{|c|}{ ER(-) } & \multirow{2}{*}{$\begin{array}{l}P \\
\text { value }^{\mathrm{a}}\end{array}$} & \multicolumn{2}{|c|}{$\mathrm{ER}(+)$} & \multirow{2}{*}{$\begin{array}{l}P \\
- \text { value }^{a}\end{array}$} & \multicolumn{2}{|c|}{$\operatorname{PR}(-)$} & \multirow{2}{*}{$\begin{array}{l}P \\
\text { value }^{a}\end{array}$} & \multicolumn{2}{|c|}{$\mathrm{PR}(+)$} & \multirow{2}{*}{$\begin{array}{l}P \\
- \text { value }^{\mathrm{a}}\end{array}$} & \multicolumn{2}{|c|}{ HER-2(-) } & \multirow{2}{*}{$\begin{array}{l}P \\
\text { value }^{\mathrm{a}}\end{array}$} & \multicolumn{2}{|c|}{ HER-2(+) } & \multirow{2}{*}{$\begin{array}{l}P \\
\text { value }\end{array}$} \\
\hline & & $\mathrm{Ca}$ & $\mathrm{OR}(95 \% \mathrm{CI})$ & & $\mathrm{Ca}$ & $\mathrm{OR}(95 \% \mathrm{CI})$ & & $\mathrm{Ca}$ & OR(95\%CI) & & $\mathrm{Ca}$ & OR(95\%CI) & & $\mathrm{Ca}$ & $\mathrm{OR}(95 \% \mathrm{CI})$ & & $\mathrm{Ca}$ & OR(95\%CI) & \\
\hline \multicolumn{20}{|l|}{ rs944289 } \\
\hline $\mathrm{CC}$ & 115 & 48 & Reference & & 79 & Reference & & 63 & Reference & & 64 & Reference & & 28 & Reference & & 99 & Reference & \\
\hline CT & 237 & 88 & $0.89(0.58,1.35)$ & 0.572 & 141 & $0.87(0.61,1.23)$ & 0.422 & 100 & $0.77(0.52,1.13)$ & 0.175 & 129 & $0.97(0.67,1.42)$ & 0.890 & 46 & $0.79(0.47,1.33)$ & 0.367 & 183 & $0.90(0.65,1.25)$ & 0.526 \\
\hline TT & 87 & 30 & $0.83(0.48,1.41)$ & 0.483 & 53 & $0.89(0.57,1.41)$ & 0.629 & 42 & $0.87(0.54,1.41)$ & 0.579 & 41 & $0.86(0.53,1.40)$ & 0.544 & 18 & $0.86(0.44,1.65)$ & 0.640 & 65 & $0.87(0.57,1.32)$ & 0.510 \\
\hline $\mathrm{CT} / \mathrm{TT}$ & 324 & 118 & $0.87(0.58,1.29)$ & 0.479 & 194 & $0.88(0.62,1.23)$ & 0.437 & 142 & $0.79(0.55,1.14)$ & 0.208 & 170 & $0.94(0.66,1.35)$ & 0.750 & 64 & $0.80(0.49,1.31)$ & 0.381 & 248 & $0.89(0.65,1.22)$ & 0.472 \\
\hline \multicolumn{20}{|l|}{ rs3787016 } \\
\hline $\mathrm{CC}$ & 149 & 49 & Reference & & 88 & Reference & & 62 & Reference & & 75 & Reference & & 31 & Reference & & 106 & Reference & \\
\hline TC & 226 & 85 & $1.19(0.79,1.80)$ & 0.404 & 124 & $0.93(0.66,1.32)$ & 0.695 & 103 & $1.14(0.78,1.67)$ & 0.495 & 106 & $0.93(0.65,1.34)$ & 0.707 & 35 & $0.72(0.42,1.22)$ & 0.224 & 174 & $1.11(0.81,1.53)$ & 0.524 \\
\hline TT & 64 & 32 & $1.53(0.90,2.63)$ & 0.119 & 61 & $1.69(1.08,2.64)$ & 0.021 & 40 & $1.51(0.91,2.49)$ & 0.108 & 53 & $1.75(1.10,2.79)$ & 0.019 & 26 & $2.10(1.14,3.88)$ & 0.017 & 67 & $1.49(0.97,2.28)$ & 0.069 \\
\hline $\mathrm{TC} / \mathrm{TT}$ & 290 & 117 & $1.24(0.84,1.82)$ & 0.287 & 185 & $1.08(0.78,1.49)$ & 0.649 & 143 & $1.20(0.84,1.72)$ & 0.324 & 159 & $1.09(0.77,1.52)$ & 0.638 & 61 & $1.00(0.62,1.60)$ & 0.986 & 241 & $1.17(0.87,1.59)$ & 0.303 \\
\hline \multicolumn{20}{|l|}{ rs1456315 } \\
\hline AA & 244 & 87 & Reference & & 147 & Reference & & 108 & Reference & & 126 & Reference & & 48 & Reference & & 186 & Reference & \\
\hline GA & 159 & 67 & $1.18(0.81,1.71)$ & 0.403 & 98 & $1.02(0.73,1.41)$ & 0.928 & 83 & $1.16(0.82,1.65)$ & 0.397 & 82 & $0.99(0.71,1.40)$ & 0.974 & 36 & $1.13(0.70,1.82)$ & 0.622 & 129 & $1.06(0.79,1.44)$ & 0.699 \\
\hline GG & 36 & 12 & $0.95(0.47,1.91)$ & 0.879 & 28 & $1.27(0.74,2.17)$ & 0.390 & 14 & $0.89(0.46,1.72)$ & 0.725 & 26 & $1.38(0.80,2.40)$ & 0.247 & 8 & $1.06(0.46,2.43)$ & 0.897 & 32 & $1.18(0.71,1.98)$ & 0.526 \\
\hline GA/GG & 195 & 79 & $1.14(0.80,1.63)$ & 0.480 & 126 & $1.06(0.78,1.44)$ & 0.696 & 97 & $1.12(0.80,1.56)$ & 0.513 & 108 & $1.07(0.78,1.47)$ & 0.691 & 44 & $1.12(0.71,1.76)$ & 0.621 & 161 & $1.09(0.82,1.44)$ & 0.575 \\
\hline \multicolumn{20}{|l|}{ rs7463708 } \\
\hline TT & 184 & 80 & Reference & & 129 & Reference & & 98 & Reference & & 111 & Reference & & 44 & Reference & & 165 & Reference & \\
\hline GT & 211 & 74 & $0.81(0.56,1.17)$ & 0.258 & 116 & $0.78(0.57,1.08)$ & 0.130 & 92 & $0.82(0.58,1.16)$ & 0.251 & 98 & $0.77(0.55,1.08)$ & 0.124 & 40 & $0.79(0.49,1.27)$ & 0.328 & 150 & $0.79(0.59,1.07)$ & 0.127 \\
\hline GG & 44 & 12 & $0.64(0.32,1.27)$ & 0.201 & 28 & $0.91(0.53,1.54)$ & 0.711 & 15 & $0.65(0.34,1.23)$ & 0.185 & 25 & $0.94(0.55,1.63)$ & 0.834 & 8 & $0.71(0.31,1.63)$ & 0.416 & 32 & $0.83(0.50,1.38)$ & 0.479 \\
\hline GT/GG & 255 & 86 & $0.78(0.54,1.11)$ & 0.170 & 144 & $0.80(0.59,1.09)$ & 0.152 & 107 & $0.79(0.56,1.10)$ & 0.162 & 123 & $0.80(0.58,1.10)$ & 0.162 & 48 & $0.78(0.50,1.22)$ & 0.278 & 182 & $0.80(0.60,1.06)$ & 0.120 \\
\hline
\end{tabular}

aAdjusted by age and menopausal status

The bold values indicate statistically significant data

To identify the stratified effects of SNPs in lncRNAs on BC risk, subgroup analysis based on the menopausal status was performed and logistic regression analysis revealed that $\mathrm{rs} 3787016 \mathrm{TT}$ genotype carriers (adjusted $\mathrm{OR}=2.55,95 \% \mathrm{CI}$ : 1.30-4.97, $P=0.006$ ) have higher $\mathrm{BC}$ risk than those with wild-type $C C$ in the premenopausal sub-cohort; in contrast, in the subgroup of postmenopausal women, rs7463708 GT genotype (adjusted OR $=0.67$,
95\% CI: $0.46-0.99, P=0.043$ ) or rs7463708 GT/GG genotype (adjusted OR $=0.68,95 \%$ CI: $0.47-0.98, P=$ 0.041 ) was associated with decreased risk of $B C$ when compared with the wild-type TT, as summarized in Table 3.

Moreover, we demonstrated the association of the four SNPs with the pathological characteristics (tumor stage, tumor grade and lymph node involvement) and patient's tumor tissue 
characteristics (expression of ER, PR and HER-2). As shown in Table 4, we found that rs3787016 TT genotype was associated with advanced TNM (III and IV) classification (adjusted OR $=2.57,95 \% \mathrm{CI}$ : 1.45-4.56, $P=0.001$ ), poor histological grade (G3-G4) (adjusted OR $=1.92,95 \%$ CI: $1.07-3.46, P=0.029$ ) and positive lymph node involvement (adjusted OR = 1.79, 95\% CI: 1.11-2.89, $P=0.016$ ). In addition, a marginal significance of increased risk for BC with early differentiation (G1-G2) (adjusted OR $=1.53,95 \%$ CI: 1.00-2.36, $P=0.053$ ) was noticed. Also, we determined a statistically significant inverse relationship between the GT genotype (adjusted OR = $0.65,95 \%$ CI: $0.43-0.97, P=0.037$ ) or GT/GG genotype (adjusted OR $=0.65,95 \%$ CI: 0.44-0.96, $P=0.029$ ) of rs7463708 polymorphism and tumor late-stage (III and IV). Furthermore, subgroup analysis based on expression of ER, PR and HER-2 was presented in the Table 5. Similarly, we observed that rs3787016 TT genotype was associated with increased BC risk of positive expression of ER (adjusted OR $=1.69,95 \% \mathrm{CI}$ : 1.08-2.64, $P=0.021$ ) and PR (adjusted OR $=1.75,95 \%$ CI: $1.10-2.79, P=0.019)$ and negative expression of HER-2 (adjusted OR $=2.10,95 \%$ CI: 1.14-3.88, $P=$ 0.017), respectively. However, there was no significant association for the three SNPs (rs944289, rs1456315, rs7463708) in all subgroups, as shown in Table 5.

\section{Discussion}

In this population-based case-control study, we investigated the association between the four selected SNPs in the lncRNAs and the risk of female BC in a Chinese population. We observed that rs3787016 TT genotype was associated with an increased risk of female BC and clinicopathologic features of the tumor, especially among premenopausal women.

The SNP rs3787016 is in a lncRNA which located in an intron region of RNA polymerase II subunit E (POLR2E) gene, which encodes the fifth largest subunit of RNA polymerase II and is responsible for synthesizing messenger RNA (mRNA) in eukaryotes. Previous study suggested the functional genetic variants in lncRNA regions may contribute to carcinogenesis [22]. Moreover, the rs3787016 TT genotype was investigated to be associated with increased risk of prostate cancer in an eastern Chinese population [23], which was consistent with the result of the study containing a meta-analysis of two GWAS and a case-control study [22]; however, such a significant association could not be duplicated in a Serbian population [24]. This study indicated that the rs3787016 TT genotype was a risk factor for female BC in a Chinese population. In contrast, a case-control study of ESCC demonstrated that POLR2E rs3787016
CT or CT/TT genotype had a decreased risk of ESCC [25]. The different findings in the above studies might be explained as follow. Firstly, the results of association studies may vary among different cancer types. Secondly, owing to ancestral backgrounds, inter-population genetic differences including differences in allele frequencies could lead to inconsistent results. Finally, ESCC is considered to be affected by multiple environmental factors exposures and the interaction of the genetic backgrounds and environmental factors contributes to the risk of cancer, so the association of genetic variants and ESCC risk should be validated by more researches. Subsequently, subgroup analysis of this study revealed that the carriers of rs3787016 TT genotype had more evident risk effect on patients with positive expression of ER and PR. As we known, the expression of these two receptors is closely related to the menopausal status of females, and women in the premenopausal status have more estrogen and progesterone, which may be attributed to the result concluded by this study that patients with rs3787016 TT genotype have higher BC risk in the premenopausal sub-cohort.

The SNP rs1456315 is located in the prostate cancer associated noncoding RNA 1 (PRNCR1), which is a $\sim 13 \mathrm{~kb}$ lncRNA transcribed from the "gene desert" region of chromosome 8q24 (128.14-128.28Mb). SNPs in the lncRNA PRNCR1 have been reported to influence the secondary structure of PRNCR1 mRNA and the stability of the mRNA conformation, resulting in the occurrence and development of human diseases [26]; in addition, rs1456315 positioned in the region 2 of $8 \mathrm{q} 24$ was significantly associated with prostate cancer susceptibility [26]. Subsequently, study reported that rs1456315 AG genotype may contribute to a decreased risk of colorectal cancer [27]. However, in the present study, no statistically significant association was observed between the rs1456315 and the risk of $\mathrm{BC}$. The inconsistent conclusions may be attributed to the different kinds of cancer. Also, this is the first study investigated the relationship between the rs1456315 and BC risk, so further large-scale studies in different populations still need to be done.

Rs7463708 overlaps with the lncRNA PRNCR1 and is located in an enhancer of prostate cancer associated transcript 1 (PCAT1) $78 \mathrm{~kb}$ away, which is a IncRNA positioned in the 8q24 "gene desert" region and overexpressed in prostate cancer. It was reported that the PCAT1 promoter strongly interacted with the $\mathrm{T}$ allele of rs7463708, suggesting that rs7463708 regulated the activation of PCAT1 enhancer and resulted in increased PCAT1 expression [28, 29]. In addition, PCAT1 plays an important role in the carcinogenesis through interacting with the GNMT 
gene involving in prostate cancer [29] and modulating mTOR signaling pathway in hepatocellular carcinoma [30], which also participating in the development of BC [31]. Thus, it is possible that the rs7463708 have potential association with BC risk. Also, our study drew a conclusion that in the sub-cohort analysis, rs7463708 GT and GT/GG genotypes were protective factors for female BC among postmenopausal status and tumor late-stage. To date, there was no study investigated the association between the rs7463708 and cancer risk except for prostate cancer risk. This is the first time investigating the association of rs7463708 and BC risk; therefore, more researches should be conducted for further study.

The SNP rs944289 at $14 \mathrm{q} 13.3$ is located $3.2 \mathrm{~kb}$ upstream of a long intergenic noncoding RNA (lincRNA) named Papillary Thyroid Carcinoma Susceptibility Candidate 3 (PTCSC3) and positioned in the binding site of the CCAAT/enhancer binding proteins (C/EBP) $\alpha$ and $\beta$ [32]. The rs944289 $\mathrm{T}$ allele can affect binding sequence and results in missense variant and amino acid substitution (valine instead of alanine at codon 339), which may be induce multinodular goiter and papillary thyroid cancer (PTC)[33]. Up to now, all published researches were assessed the relationships between the rs944289 and differentiated thyroid carcinoma (DTC) [34-37]; however, to date, no association of other cancer types was discussed with the SNP, which may be due to the specific susceptibility of the SNP to the risk of DTC, and in this study, we also observed no significant relationships between the rs 944289 and BC risk.

To our knowledge, in the present study, we investigated the association between the four selected SNPs and BC risk for the first time. Although there were some important discoveries revealed in the study, several limitations also need to be addressed. Firstly, the four selected SNPs of IncRNAs in our study may not be comprehensive because we were limited by those have been identified to have risk effect on other cancers. Also, the biological function of these lncRNAs remains largely unknown and has not been validated in experimental models, so it is difficult to explain our results. Secondly, the number of subjects in our study is not enough large and the small size in subgroup analysis may not provide statistical power to show significant results. Moreover, the clinical information of each individual is not fully reliable and detailed, which might influence the accuracy of the results. Thirdly, BC is a complex and multifactorial disease, this is not a large size population based case-control study, and the samples was not enough for the sub-group analysis, therefore, to confirm our findings, studies with more large-scale samples including different ethnic populations and detailed clinical information should be conducted. In addition, properly functional assessments also should be performed to illuminate the etiology of the BC.

In summary, this study demonstrated that rs3787016 TT genotype was associated with BC risk and clinicopathologic features of the tumor, especially among premenopausal women. Nevertheless, the results of this preliminary study need to be validated by further larger and well-designed researches.

\section{Acknowledgments}

This project was supported by grants from the National Nature Science Foundation of China (No.81472027 to S.W and No.81501820 to Y.P); Jiangsu Provincial Medical Youth Talent to B.H (QNRC2016066) and Y.P (QNRC2016074); Nanjing Medical Science and Technique Development Foundation to B.H (JQX13003) and Nanjing Medical University Science and Technique Development Foundation Project to HL. S (No.2015NJMUZD049).

\section{Competing Interests}

The authors have declared that no competing interest exists.

\section{References}

1. Torre LA, Bray F, Siegel RL, et al. Global cancer statistics, 2012. CA: a cancer journal for clinicians. 2015; 65: 87-108.

2. Chen W, Zheng R, Baade PD, et al. Cancer statistics in China, 2015. CA: a cancer journal for clinicians. 2016; 66: 115-32.

3. Michailidou K, Beesley J, Lindstrom S, et al. Genome-wide association analysis of more than 120,000 individuals identifies 15 new susceptibility loci for breast cancer. Nature genetics. 2015; 47: 373-80.

4. Sawyer E, Roylance R, Petridis C, et al. Genetic predisposition to in situ and invasive lobular carcinoma of the breast. PLoS genetics. 2014; 10: e1004285.

5. Fanale D, Amodeo V, Corsini LR, et al. Breast cancer genome-wide association studies: there is strength in numbers. Oncogene. 2012;31: 2121-8.

6. Mattick JS. The functional genomics of noncoding RNA. Science. 2005; 309: 1527-8.

7. Li L, Feng T, Lian Y, et al. Role of human noncoding RNAs in the control of tumorigenesis. Proceedings of the National Academy of Sciences of the United States of America. 2009; 106: 12956-61.

8. Zhang X, Rice K, Wang Y, et al. Maternally expressed gene 3 (MEG3) noncoding ribonucleic acid: isoform structure, expression, and functions. Endocrinology. 2010; 151: 939-47.

9. Gupta RA, Shah N, Wang KC, et al. Long non-coding RNA HOTAIR reprograms chromatin state to promote cancer metastasis. Nature. 2010; 464: 1071-6.

10. Moran VA, Perera RJ, Khalil AM. Emerging functional and mechanistic paradigms of mammalian long non-coding RNAs. Nucleic acids research. 2012; 40: 6391-400.

11. Ji P, Diederichs S, Wang W, et al. MALAT-1, a novel noncoding RNA, and thymosin beta4 predict metastasis and survival in early-stage non-small cell lung cancer. Oncogene. 2003; 22: 8031-41.

12. Peng $\mathrm{W}, \mathrm{Si} \mathrm{S}$, Zhang $\mathrm{Q}$, et al. Long non-coding RNA MEG3 functions as a competing endogenous RNA to regulate gastric cancer progression. Journal of experimental \& clinical cancer research : CR. 2015; 34: 79.

13. Burd CE, Jeck WR, Liu Y, et al. Expression of linear and novel circular forms of an INK4/ARF-associated non-coding RNA correlates with atherosclerosis risk. PLoS genetics. 2010; 6: e1001233.

14. Zhang X, Zhou L, Fu G, et al. The identification of an ESCC susceptibility SNP rs920778 that regulates the expression of lncRNA HOTAIR via a novel intronic enhancer. Carcinogenesis. 2014; 35: 2062-7.

15. Guo L, Lu X, Zheng L, et al. Association of Long Non-Coding RNA HOTAIR Polymorphisms with Cervical Cancer Risk in a Chinese Population. PloS one. 2016; 11: e0160039.

16. Bayram S, Sumbul AT, Batmaci CY, et al. Effect of HOTAIR rs 920778 polymorphism on breast cancer susceptibility and clinicopathologic features 
in a Turkish population. Tumour biology : the journal of the International Society for Oncodevelopmental Biology and Medicine. 2015; 36: 3863-70.

17. Liu Y, Pan S, Liu L, et al. A genetic variant in long non-coding RNA HULC contributes to risk of HBV-related hepatocellular carcinoma in a Chinese population. PloS one. 2012; 7: e35145.

18. $\mathrm{Li} \mathrm{N}$, Zhou P, Zheng J, et al. A polymorphism $\mathrm{rs} 12325489 \mathrm{C}>\mathrm{T}$ in the lincRNA-ENST00000515084 exon was found to modulate breast cancer risk via GWAS-based association analyses. PloS one. 2014; 9: e98251.

19. Gabriel S, Ziaugra L, Tabbaa D. SNP genotyping using the Sequenom MassARRAY iPLEX platform. Current protocols in human genetics / editorial board, Jonathan L Haines [et al]. 2009; Chapter 2: Unit 212.

20. Thomas RK, Baker AC, Debiasi RM, et al. High-throughput oncogene mutation profiling in human cancer. Nature genetics. 2007; 39: 347-51.

21. Gul AE, Keser $\mathrm{SH}$, Barisik NO, et al. The relationship of cerb $\mathrm{B} 2$ expression with estrogen receptor and progesterone receptor and prognostic parameters in endometrial carcinomas. Diagnostic pathology. 2010; 5: 13.

22. Jin G, Sun J, Isaacs SD, et al. Human polymorphisms at long non-coding RNAs (lncRNAs) and association with prostate cancer risk. Carcinogenesis. 2011; 32: $1655-9$.

23. Cao DL, Gu CY, Zhu Y, et al. Polymorphisms at long non-coding RNAs and prostate cancer risk in an eastern Chinese population. Prostate cancer and prostatic diseases. 2014; 17: 315-9.

24. Nikolic ZZ, Brajuskovic GN, Pavicevic D, et al. Assessment of possible association between rs3787016 and prostate cancer risk in Serbian population. International journal of clinical and experimental medicine. 2013; 6: 57-66.

25. Kang M, Sang Y, Gu H, et al. Long noncoding RNAs POLR2E rs3787016 C/T and HULC rs7763881 A/C polymorphisms are associated with decreased risk of esophageal cancer. Tumour biology : the journal of the International Society for Oncodevelopmental Biology and Medicine. 2015; 36: 6401-8.

26. Chung $S$, Nakagawa $H$, Uemura $M$, et al. Association of a novel long non-coding RNA in 8q24 with prostate cancer susceptibility. Cancer science. 2011; 102: 245-52.

27. Li L, Sun R, Liang $\mathrm{Y}$, et al. Association between polymorphisms in long non-coding RNA PRNCR1 in 8q24 and risk of colorectal cancer. Journal of experimental \& clinical cancer research : CR. 2013; 32: 104

28. Guo H, Ahmed M, Zhang F, et al. Modulation of long noncoding RNAs by risk SNPs underlying genetic predispositions to prostate cancer. Nature genetics. 2016.

29. [No authors listed]. A Prostate Cancer Risk SNP Upregulates the Tumorigenic IncRNA PCAT1. Cancer discovery. 2016.

30. Yen $\mathrm{CH}, \mathrm{Lu} \mathrm{YC}, \mathrm{Li} \mathrm{CH}$, et al. Functional characterization of glycine $\mathrm{N}$-methyltransferase and its interactive protein DEPDC6/DEPTOR in hepatocellular carcinoma. Molecular medicine. 2012; 18: 286-96.

31. Wazir U, Wazir A, Khanzada ZS, et al. Current state of mTOR targeting in human breast cancer. Cancer genomics \& proteomics. 2014; 11: 167-74.

32. Jendrzejewski J, He H, Radomska HS, et al. The polymorphism rs 944289 predisposes to papillary thyroid carcinoma through a large intergenic noncoding RNA gene of tumor suppressor type. Proceedings of the National Academy of Sciences of the United States of America. 2012; 109: 8646-51.

33. Gao Y, Chen F, Niu S, et al. Replication and Meta-Analysis of Common Gene Mutations in TTF1 and TTF2 with Papillary Thyroid Cancer. Medicine. 2015; 94: e1246.

34. Tcheandjieu C, Lesueur F, Sanchez M, et al. Fine-mapping of two differentiated thyroid carcinoma susceptibility loci at 9q22.33 and $14 \mathrm{q} 13.3$ detects novel candidate functional SNPs in Europeans from metropolitan France and Melanesians from New Caledonia. International journal of cancer. 2016; 139: 617-27.

35. Pereda CM, Lesueur F, Pertesi M, et al. Common variants at the $9 \mathrm{q} 22.33$, $14 \mathrm{q} 13.3$ and ATM loci, and risk of differentiated thyroid cancer in the Cuban population. BMC genetics. 2015; 16: 22.

36. Maillard S, Damiola F, Clero E, et al. Common variants at 9q22.33, 14q13.3, and ATM loci, and risk of differentiated thyroid cancer in the French Polynesian population. PloS one. 2015; 10: e0123700.

37. Wang YL, Feng SH, Guo SC, et al. Confirmation of papillary thyroid cancer susceptibility loci identified by genome-wide association studies of chromosomes 14q13, 9q22, 2q35 and 8p12 in a Chinese population. Journal of medical genetics. 2013; 50: 689-95. 